\section{P1-14 IDENTIFYING ADVERSE EVENTS OF VACCINES USING A BAYESIAN METHOD OF MEDICALLY GUIDED INFORMATION SHARING}

doi:10.1136/jech.2011.142976c.8

${ }^{1} \mathrm{C}$ Crooks, ${ }^{*} \mathrm{D}$ Prieto-Merino, ${ }^{2} \mathrm{~S}$ Evans. ${ }^{1}$ University of Nottingham, Nottingham, UK; ${ }^{2}$ London School of Hygiene and Tropical Medicine, London, UK

Introduction The detection of adverse events following immunisation (AEFI) fundamentally depends on how these events are classified. Standard methods impose a choice between either grouping similar events together to gain power or splitting them into more specific definitions. We demonstrate a method of medically guided Bayesian information sharing that avoids grouping or splitting the data, and we further combine this with the standard epidemiological tools of stratification and multivariate regression.

Methods All spontaneous reports of gastrointestinal AEFI in children under 18 years old in the WHO (Uppsala Monitoring Centre) Vigibase $($ were used to calculate reporting ORs for each AEFI and vaccine combination. After testing for effect modification these were then reestimated using multivariable logistic regression adjusting for age, gender, year and country of report. A medically guided hierarchy of AEFI terms was then derived to allow information sharing in a Bayesian model.

Results A crude analysis identified 132 signals from 655 reported combinations of gastrointestinal AEFI. Adjusting for confounding, where appropriate, reduced the number of signals identified to 88 . The addition of a Bayesian hierarchical model identified four further signals and removed three. Effect modification by age and gender was identified for six vaccines.

Conclusion This study demonstrated a sequence of methods for routinely analysing spontaneous report databases that was easily understandable and reproducible. The combination of classical and Bayesian methods in this study help to focus the limited resources for hypothesis testing studies towards the adverse events with the strongest support from the data.

\section{P1-15 OBESITY CLUSTERING IN CEBU, PHILIPPINES: AN APPLICATION OF SATSCAN AND THE SPATIAL SCAN STATISTIC}

doi:10.1136/jech.2011.142976c.9

D Dahly. ${ }^{*}$ University of Leeds, Leeds, UK

Introduction Obesity is an important, global, public health problem. To promote prevention, more research is needed to understand exactly how shared environments impact obesogenic behaviours. Identifying spatial clusters of obesity is the first step towards a better understanding of its environmental drivers, and can immediately inform public health practice. To date, research has overlooked lower-income contexts, where obesity is emergent and environments are changing at an unprecedented pace.

Methods Using data from a cohort of young adult Filipinos $(21.5 \mathrm{y}$; $n=1808$ ), we used the Kulldorff spatial scan statistic to detect areas in Metropolitan Cebu with a high sample prevalence of obesity. Cluster locations were then compared to the urbanicity of constituent neighbourhoods. We also tested whether clusters were explained by the spatial distribution of household-assets scores in the study participants.

Results Significantly unusual clusters (rejection of $\mathrm{H} 0$ : complete spatial randomness, at $\mathrm{p}<0.05$ ) of overweight and obesity were detected for males and females. Clusters were primarily located in urban areas, but typically extended into peri-urban and even rural neighbourhoods. The exact location of clusters varied as a function of both sex and measure of obesity used. Clusters in males, but not females, were explained by the spatial distribution of socioeconomic status.

Conclusions Where a young adult lives is a strong predictor of obesity risk in Cebu. Environmental drivers of obesity among young adults in Cebu may vary by gender. Using simple urban-rural classifications to contextualise obesity in lower income countries may be overly simple, and misdirect public health efforts.

\section{P1-16 A LATENT CLASS ANALYSIS OF SOCIOECONOMIC STATUS AND OBESITY IN YOUNG ADULTS FROM CEBU, PHILIPPINES}

doi:10.1136/jech.2011.142976c.10

D Dahly, ${ }^{*}$ M Gilthorpe. University of Leeds, Leeds, UK

Introduction Socioeconomic status (SES) is a critical driver of human health, but in research practice it is rarely well-defined and inconsistently measured. Latent class analysis (LCA) is a potentially useful method of characterising SES, particularly when multiple SES indicators are available. We employed LCA to better understand how SES is related to obesity in a sample of young Filipino adults; and contrasted LCA with other approaches.

Methods Data are from a cohort of young adults enrolled in the Cebu Longitudinal Health and Nutrition Survey $(987$ males and 819 females). Latent classes were derived using Mplus mixture modelling. Class indicators included obesity status, marital status, education level, urbanicity, household assets and income. Models were estimated under the assumption of class-conditional independence, with no further parameter constraints.

Results For both sexes, a 3-class solution was the best balance of model fit (using log-likelihood, AIC, and BIC) and parsimony. Overall obesity prevalence was $9.4 \%$ in males and $7.8 \%$ in females. One class of males $(n=194)$ had an obesity prevalence of $22 \%$, vs $6 \%$ in the remaining two classes ( $p=0.007$ for $\mathrm{H} 0$ : no difference). They were more likely to be urban, educated, and unmarried than other males $(p<0.05)$. However, a female class $(n=257)$ with a similar socioeconomic profile instead had the lowest prevalence of obesity (5.5\%).

Conclusions LCA can contribute to our understanding of socioeconomic drivers of health. Interpretation of LCA is discussed in the context of Rothman and Greenland's model of causation.

\section{P1-17 THE TREELET TRANSFORM-A NOVEL METHOD FOR DETERMINING PATTERNS IN ADIPOSE TISSUE FATTY ACIDS}

doi:10.1136/jech.2011.142976c.11

${ }^{1,2} \mathrm{C} C$ Dahm, ${ }^{*}{ }^{1,2} \mathrm{~J} N$ Østergaard, ${ }^{1,3} \mathrm{~A}$ Gorst-Rasmussen, ${ }^{2} \mathrm{M}$ U Jakobsen, ${ }^{1} \mathrm{E}$ B Schmidt, ${ }^{4} \mathrm{~A}$ Tjønneland, ${ }^{2} \mathrm{~K}$ Overvad. ${ }^{1}$ Department of Cardiology, Center for Cardiovascular Research, Aalborg Hospital, Aarhus University Hospital, Aalborg, Denmark; ${ }^{2}$ Department of Epidemiology, School of Public Health, Aarhus University, Aarhus, Denmark; ${ }^{3}$ Department of Mathematical Sciences, Aalborg University, Aalborg, Denmark; ${ }^{4}$ Institute of Cancer Epidemiology, Danish Cancer Society, Copenhagen, Denmark

Introduction Dietary fatty acid intake may be associated with risk of obesity and non-communicable disease. Adipose tissue fatty acids are correlated, reflecting shared dietary sources and metabolic processes. To date fatty acids have been investigated individually, or using principal component analysis (PCA), but interpretation of such studies is not trivial. The treelet transform (TT) is a novel method for generating sparse factors that describe the correlation structure of the data. In studies of dietary patterns TT is as efficient in extracting factors as PCA, and simpler to interpret. We therefore compared factors determined by PCA and by TT to evaluate interpretability of patterns in adipose tissue fatty acids. 
Methods 34 fatty acids from adipose tissue biopsies were determined in a random sample of 1100 men and women from the Diet, Cancer and Health study. PCA and TT were conducted on the fatty acid data correlation matrix. The stability of the analyses was evaluated, and the highest variance factors were extracted and descriptively compared.

Results TT factors consisted of distinct groupings of 3-8 fatty acids, generally characterised by hydrocarbon chain length and saturation status. PCA factors consisted of complex weightings of all 34 fatty acids, where some fatty acid groupings loaded strongly on some factors.

Conclusions Fatty acid patterns determined using TT are considerably simpler to interpret than those generated by PCA, an advantage in studies of the effects of complex multidimensional exposures. Future work will relate these patterns to risk of disease.

\section{P1-18 STUDYING EARLY PREGNANCY DURING INFERTILITY TREATMENT MAY IDENTIFY NOVEL RISK FACTORS FOR CONGENITAL MALFORMATIONS}

doi:10.1136/jech.2011.142976c.12

M Davies, ${ }^{*} \mathrm{~K}$ Willson, V Moore. University of Adelaide, Adelaide, South Australia, Australia

Introduction The study of early pregnancy risk is generally very difficult, but potentially feasible in women during clinical infertility treatment with gamete and embryo data, known gestation, routine ultrasound in early pregnancy, and detailed recording of birth outcomes.

Methods All treatment cycles of assisted reproductive technology (ART) for the period January 1986 to December 2002 in South Australia were linked to both the routine State perinatal collection and the registries for birth defects and cerebral palsy (coded to ICD-9 BPA). Fetal loss was assessed by comparing routine 6 week ultrasound data and babies delivered. ORs for birth defects were calculated for deliveries with an empty fetal sac at 6 weeks, or subsequent fetal loss and a baby delivered, compared to singleton pregnancies without loss.

Results The prevalence of congenital malformations was $14.6 \%$ in pregnancies in which there had been an empty sac at a 6 week ultrasound. The presence of an empty sac was associated with both an increased risk of any malformation $(\mathrm{OR}=1.93$, CI 1.10 to 3.39) and with multiple malformations $(\mathrm{OR}=2.78$, CI 1.27 to 6.03). Multiple pregnancy without fetal loss was not associated with an overall increased prevalence of malformation $(\mathrm{OR}=1.01$, CI 0.81 to 1.25 ).

Conclusions The presence of an empty fetal sac at 6 weeks gestation constitutes a significant risk factors for congenital malformations in the surviving baby. Subsequent work identifying upstream factors influencing embryo development and loss have significant potential for advancing our understanding of the aetiology of congenital malformations, particularly after infertility treatment.

\section{P1-19 THE DEVELOPMENT OF ETHNIC-SPECIFIC FOOD FREQUENCY QUESTIONNAIRES (FFOS) TO MEASURE DIET OF NON- WESTERN MIGRANTS IN THE NETHERLANDS}

doi:10.1136/jech.2011.142976c.13

\footnotetext{
${ }^{1,2} \mathrm{M}$ Beukers, ${ }^{1} \mathrm{~L}$ Dekker, ${ }^{3} \mathrm{~J}$ de Vries, ${ }^{2} \mathrm{H}$ Brants, ${ }^{2} \mathrm{E}$ de Boer, ${ }^{3} \mathrm{C}$ Perenboom, ${ }^{1} \mathrm{M}$ Snijder, ${ }^{1} \mathrm{~K}$ Stronks, ${ }^{1} \mathrm{M}$ Nicolaou. ${ }^{1}$ Department of Public Health, Academic Medical Center, University of Amsterdam, Amsterdam, The Netherlands, ${ }^{2}$ Centre for Nutrition
}

and Health, Institute for Public Health and the Environment, Bilthoven, The Netherlands, ${ }^{3}$ Division of Human Nutrition, Wageningen University, Wageningen, The Netherlands

Introduction Diet is an important modifiable risk factor for cardiovascular disease and appears relevant in migrant groups in Western Europe, including the Netherlands. However, no comprehensive picture of the dietary patterns of the main non-western migrants in the Netherlands exists. Research is limited by a lack of validated instruments to measure habitual diet. In this study we aimed to develop ethnic-specific FFOs in order to study the dietary patterns of Surinamese of African and of South Asian origin, Turkish and Moroccan individuals residing in Amsterdam, the Netherlands.

Methods Food items were selected according to their percentage contribution to the nutrients of interest based on data from $24 \mathrm{~h}$ recalls. Tests of face-validity and cognitive interviews were performed to pinpoint problems in design and comprehension of the FFQs. A nutrient database was constructed based on data in the Dutch Food Composition Table.

Results Three FFQs including 180-200 food items have been developed to reflect usual intakes of Turkish, Moroccan and Surinamese migrants. Overall the FFOs cover more than $94 \%$ of the intake of the nutrients at interest in this study.

Conclusion With the development of the ethnic-specific FFOs, this study provides an opportunity to move the field of nutritional and health epidemiology forward. The FFOs will be applied to participants in the HELIUS study, a multi-ethnic cohort in Amsterdam, and will enable us to gather dietary intake data of 1000 participants (18-70 year old) per ethnic group. This will allow research into the main determinants and health consequences of habitual diet.

\section{P1-20 EARLY SCREENING FOR CORONARY ARTERY DISEASE IS NEEDED IN SOUTH ASIAN INDIAN IMMIGRANTS WITH TYPE 2 DIABETES}

doi:10.1136/jech.2011.142976c.14

${ }^{1} \mathrm{~S}$ Dodani, ${ }^{*} \mathrm{G}$ Sharma. ${ }^{1}$ University of Kansas Medical Center, Leawood, USA; ${ }^{2}$ Medical College of Georgia, Augusta, USA

Background South Asian Immigrants (SAIs) are the second fastest growing Asian immigrant population in the USA, and at a higher risk of type 2 diabetes (diabetes) than the general USA population. Coronary Artery Disease (CAD) is the principal cause of mortality globally, particularly in diabetic subjects. In this study, we sought to determine the; (1) Distribution of risk factors for $\mathrm{CAD}$ in diabetic and non-diabetic SAIs; and (2) Presence of sub-clinical CAD in diabetic and non diabetic SAIs in the USA.

Methods 213 first generation SAIs subjects were recruited and broadly divided into two subgroups; 35 diabetics and 178 non diabetics. Their risk factors for CAD were compared. For sub-clinical CAD assessment, Common Carotid Artery Intima-Media Thickness (CCA-IMT) was used as a surrogate marker for atherosclerosis. For CAD diagnosis, Exercise Tolerance stress Test (ETT) was performed. Results Both diabetics and non diabetics SAIs in general, share a very heavy burden of CAD risk factors. Hypertension $(p=0.003)$, high cholesterol $(p<0.0001)$ and family history of diabetes $(p<0.0001)$ was significantly associated with diabetes. Presence of sub-clinical CAD was also higher in diabetics as compared to non diabetics (63\% vs $52 \%$ ). $45 \%$ of diabetics (who were not previously diagnosed with $\mathrm{CAD}$ ) were found to be ETT positive for CAD $(\mathrm{p}<0.0001)$

Conclusion $\mathrm{CAD}$ risk factors and sub-clinical CAD are more prevalent among diabetic SAIs. Early screening and aggressive treatment for risk factor reduction in SAIs is the key to combating the increasing incidence of $\mathrm{CAD}$. Larger prospective trials are required to confirm these study findings. 